\title{
Extracellular matrix and its role in conveying glial/neural interactions in health and disease
}

\author{
Vera Maura Fernandes de Lima ${ }^{\mathrm{a}, \mathrm{b}, *}$ and Wolfgang Hanke ${ }^{\mathrm{c}}$

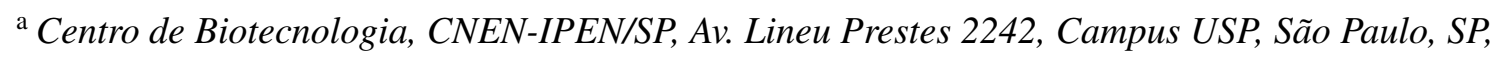 \\ Brazil, 05508-000 \\ ${ }^{\mathrm{b}}$ LIM-26 Faculdade de Medicina da USP-SP, São Paulo, Brazil \\ ${ }^{\mathrm{c}}$ Membrane Physiology Division, Institute of Physiology 230, Hohenheim University, Stuttgart, \\ Germany
}

Received 1 December 2016

Accepted 9 January 2017

\begin{abstract}
We review the concepts and findings that may be related to the occurrence of non-linear glial/neural dynamics involving interactions between polyelectrolytes of the extracellular matrix and the basement membranes that cover the endfeet of glia at CNS interfaces. Distortions of perception and blocking of learning expressed in functional syndromes are interpreted as macroscopic electrochemical patterns that emerge in grey matter through glial/neural interactions.
\end{abstract}

Keywords: Extracellular matrix, polyelectrolytes, excitable media, cognition, migraine, epilepsy, smart gels, volume phase transitions

\section{Introduction}

The year was 1940. The neuroscientist Karl Lashley, a migraine sufferer, thought deeply about the march of scotomas of the migraine aura and reached two conclusions. 1 - features of functional syndromes could teach us how the brain works; 2 - the scotomas of the migraine aura were the perceptual counterpart of an excitation/inhibition wave spreading at $3 \mathrm{~mm} / \mathrm{min}$ constant velocity through the primary visual cortex V1 (Lashley [39]). Unbeknownst to Lashley, a young PhD student at Harvard named Aristides Leão, using a new (Grass) EEG machine, discovered in rodent cortices a wave of sudden potential drop followed by a depression of the EEG electrical oscillations. The wave he observed spread from the occipital pole to the central gyrus of the smooth surface of the rodent cortex with a velocity of $3 \mathrm{~mm} / \mathrm{min}$. As with action potentials, the wave amplitude remained constant as it spread. Leão realized that such a wave occurring in the primary somatosensory cortex of humans could explain features of the spread of motor Jacksonian seizures for example. His discovery, published in 1944 (Leão [40]), shows

\footnotetext{
*Corresponding author. E-mail: vmflima@ipen.br.
} 
careful observation of phenomena accompanying the initial potential drop; for example, dilation of pial blood vessels signaling an increase in the metabolic demands of tissue following the wave onset.

If Lashley and Leão had been physicists rather than 1940s neuroscientists, they might soon have become aware of their separate discoveries and a new sub-field of research could have been born: one concerned with excitation waves in the brain. Lashley's understanding of the issues is clear from the title of his paper: "Patterns of cerebral integration indicated by the scotomas of migraine" (Lashley [39]). Leão's perceptiveness is shown in his discussion of implications of his discovery. Sadly they were not physicists but belonged to what was then the more disorganized field of biology. It took 18 years for anyone to suggest matching the migraine aura perceptual experience with Leão's spreading depression wave (Milner [43]).

In this paper, we will argue that a range of macroscopic electrochemical patterns emerge in grey matter through glial/neural interactions that depend on the polyelectrolyte gel of the extracellular matrix. This charged gel is a physical-chemical medium very different from ordinary dilute ion solutions; when the gel undergoes a phase transition, so too does the related tissue dynamics. Our account starts with some of the history of ideas about glial neural interactions and of our knowledge about the properties of excitable media.

\section{The glial/neural dynamics brain and the spreading depression wave}

Santiago Ramon y Cajal had his neuron doctrine ready by 1891 (Guillery [28]). He postulated that neurons are the fundamental functional 'units' of the brain. In 1896 an alternative view of how the brain works was published by Carl Ludwig Schleich (Dierig [9]) proposing a relationalist view of brain function dependent on glial/neural interactions. Dierig's [9] paper reproduces two of Schleich's original drawings, which picture glia as the only element present at the pial interface of the cortex and show a good intuitive feeling for what we now know is the actual glial/neural membrane area ratio in the cortex.

Cajal's doctrine was hierarchical, cortical neurons at the top of the control, a view not shared by Camillo Golgi, the inventor of the cell staining technique mastered by Cajal. In 1906, a network or relationalist view of the brain was defended by Golgi. In that year, both scientists shared the Nobel Prize for Physiology and Medicine. Thus just at the beginning, the two views of the CNS were blessed with the same award.

The next landmark in this story is contained in Robert Galambos' review (Galambos [20]). In an autobiographical chapter, he describes the original insight leading to, and the consequences for his career, of his remarkable paper (Galambos [21]): "on an airplane somewhere between Chicago and the Grand Canyon, I turned to my companion, Harvey Savely, and announced: I know how the brain works, and for the next hour or so, bent his ear with the ideas published two months later in the paper "A GliaNeuron theory of brain function" (Galambos [21]). In his review Galambos assumed that in the central grey matter, all the cells that were not neurons, were glial cells. Here we will modify this concept a bit: all cells that are not neurons have glial cell function. One example: tanycytes are a type of ependymal cells that make the interface of the third ventricle, here we assume that both the ependymal and tanycyte populations are glial cells, and indeed tanycytes express the hallmark of glia, the Glial Fibrillary Acidic Protein (GFAP).

Galambos was especially concerned with the spreading depression wave as a phenomenon not readily explained in terms of neural theory. He proposed that, if the then recent findings of Tasaki and Chang 
(Tasaki and Chang [69]) were taken into account, a glial origin for the slow potential drop at the spreading depression wave onset might provide a good explanation for the wave's macroscopic concomitant. Galambos also called attention to the fact that spreading depression blocked learned behavior in animals.

In the subsequent decade Galambos' prediction was validated by a group headed by Tetsuo Tomita who described relevant findings in in vitro retinas and another group who described the glial depolarization associated with waves in the cortex (Sugaya et al. [62]; Mori et al. [45-47]; Tomita [72]). In the cat cortex, Sugaya et al. [62] showed with intracellular electrodes that glial membrane depolarization coincided with the extracellular potential drop measured with surface electrodes ( 93 cells from 35 animals). In 15 cells localized from 100 to $3000 \mu \mathrm{m}$ deep within the cortex, the potential recorded extracellularly from the glial cell changed in the opposite direction to the simultaneously recorded glial depolarization. The neural response was more variable than the glial one. The most probable response of nearby neurons was a short burst of action potentials at wave onset followed by silence over the next minute. That's true for all central grey matter. Tomita's group found that the extracellular potential drop also coincided with glial depolarization and that at the outer retina, horizontal cells still responded to light while the potential drop of the wave was evolving. In the retina, the peak of the extracellular $d V / d t$ also coincides with the maximum rate of change of the optical properties in nearby tissue, interpreted as structural changes in glia (Fernandes de Lima et al. [15]). The spreading depression wave is the result of membrane interactions between glia and neurons that can only happen through the extracellular matrix.

Galambos addressed one more time the role of glia in the brain in his 2007 [22] paper (he died in 2010). In this paper he no longer makes any mention of either Ichigi Tasaki's discovery of cortical potentials and glial cell activity (by now Tasaki was a full-fledged heretic banished from mainstream neuroscience journals) as well as of the spreading depression wave. He waived his 1961 prediction that glial membrane depolarization could explain the potential drop of wave onset. He instead called attention to the concept of a tripartite synapse that had been proposed by Araque et al. [1]. And he criticized it as an oversimplification of the range of possible membrane interactions at a central synapse - i.e. neuronneuron, glia-glia, glia-neuron and neuron-glia, each with a two-way possibility. It should be noted that, at the neuromuscular junction, the tripartite model fits well. In the 1961 review, Galambos also commented on the results of Birks et al. [2] that showed direct glia-muscle stimulation following axonal degeneration in the neuromuscular junction. Our group at Hohenheim and São Paulo (USP) universities demonstrated glial initiation of excitation in central grey matter unequivocally in in vitro retinas using hydrophilic proteins, and also a high probability of initiation of excitation using the poisonous glycoside ouabain (also large and hydrophilic). We refer to the results of exposing the inner limiting membrane layer to gyroxin, protamine, crotamine and ouabain (da Silva et al. [7]; Fernandes de Lima et al. [13,16]). We could see excitation spread with latencies at millisecond range (gyroxin and crotamine). Putting these results together, we can say that the basement membrane endfeet layer (or glial membrane) can initiate responses that change the whole tissue state and, in awake animals, may elicit seizures or complex sequences of motor behavior. The description of Lashley was correct, the migraine aura is an excitation wave (the glial depolarization phase-order of seconds) followed by whole tissue inhibition (time scale of one to two minutes).

It appears that Galambos' insight was correct and, if we want to understand how the brain works, we should look at how the glial network controls neural input and output. The relationalist brain of Golgi appears better suited to explain perception than the neuron-as-unit doctrine of Cajal, since in it there are no units but only networks of relations. 


\section{The basement membrane and extracellular matrix are both excitable media}

In the Introduction we pointed out that, like action potentials, spreading depression waves propagate with uniform amplitude. This property is not the only one shared with action potentials: both have a threshold, a wave of excitation followed by refractory periods before recovery of full excitability, and both annihilate if separate events collide. They are similar because they belong to the same class of electrochemical phenomena; both are examples of active wave propagation in excitable media. They differ in spatial dimensionality: one, along the axon membrane, in the case of action potentials, but two (or close enough) for spreading depression waves in the thin retina ( $\sim 250 \mu$ m maximum width). Heartbeats, on the other hand, are manifestations of three dimensional scroll excitation waves. They are all macroscopic patterns expressed at the supramolecular and supracellular level (see Fernandes de Lima and Hanke [14]). Brain and heart, if viewed as excitable media, must share similar rules for selforganization of spatial and temporal patterns.

Vladimir Zykov [79] wrote, in an excellent account of excitable media: "In an excitable medium it is possible for excitation to be passed from one segment to another by means of local coupling. Thus, an excitable medium is able to support propagation of undamped solitary excitation waves, as well as wave trains"... In the meeting referred to below (footnote 1), the west got acquainted with the most successful experimental model of dissipative structures, the Belouzov-Zabotinsky (B-Z) reaction system. It provides a useful model of the relevant dynamics which can express temporal oscillations and/or self-organized spatial waves depending on experimental context.

According to the excitable media interpretation, it is likely that a wide range of phenomena, including the EEG alpha rhythm and the potential drop at the onset of the spreading-depression waves, are expressions of a dynamics involving glial-neural interactions through the extracellular matrix. In the B-Z case the analogous dynamics depends on an autocatalytic reaction modelled by non-linear quadratic or cubic expansive terms, modified by relatively slow, linear inhibition. In this paper the authors adopt the Ichigi Tasaki excitable membrane model (see Appendix) and as such the adequate mathematical model is the activator-inhibitor model of FitzHugh-Nagumo (see Tasaki [64,65]). According to Vladimir Zykov, the brusselator and the FitzHugh-Nagumo equations show mathematical identity. This mathematical identity of their dynamics, we thought, explained the parallel behavior of the two systems' responses to manipulations of weak external forces (gravity and electromagnetic forces, see Hanke et al. [29] and Wiedemann et al. [75]).

The field that now is called excitable media, is probably the happy result of the fast growth of computer power in the late sixties and seventies of last century as well as of a population of applied mathematicians, together with suitable experimental models and the modelling of their behavior in computers and finally, a theoretical breakthrough in irreversible thermodynamics. Until very recently, we would have traced the birth of the excitable media field back to Prigogine's publication of his brusselator model (Prigogine and Lefevre [52]) and to an historical meeting between Eastern and Western scientists in Prague, ${ }^{1}$ both events occurring in 1968. In 1971 Prigogine publishes his famous minimum entropy production theorem (Glansdorff and Prigogine [24]). However we subsequently became aware of the work

\footnotetext{
${ }^{1}$ In 1968 the president of the Czechoslovakian Academy of Science was Jan Bures, a leader in the experimental spreading depression. In that year, a Symposium was held at the Academy reuniting East and West scientists during the Cold War, a rare event. In this meeting, the young Anatol Zabotinsky showed his experiments with the B-Z system. Bures immediately realized that the spreading depression and B-Z waves were related phenomena and he predicted and later showed the existence of two dimensional spiral waves in in vitro retinas (Gorelova and Bures [25]). [From personal communication to authors by Jan Bures and Hiss Martins-Ferreira.]
} 
of Motoyosi Sugita, a Japanese physicist who worked alone in post-war Japan and, using a different formalism, arrived at similar conclusions as Prigogine's at least ten years earlier (see Iguchi [33]). In his analysis of transient phenomena that we now call steady state far from equilibrium, Sugita proposed that the dissipation of free energy (Gibb's free energy) is what maintains the structures that Ilya Prigogine referred to as dissipative structures. However, Sugita went beyond Prigogine. He proposed that the dissipation rate tends to increase, or the rate of change of energy dissipation tends to a maximum, in dissipative structures. He called this principle the fourth law of thermodynamics. In his view, all life depended on this fourth law.

A turning point in our understanding of the parallelism between $\mathrm{B}-\mathrm{Z}$ and retinal wave behavior, came in 2009 with the use of liquid deuterium in place of water as the solvent in both systems. The idea was to modify a component of the global coupling in the systems, the solvent. The physical properties of deuterium solutions differ from those of water solutions, as shown by John Katz in 1960 (Katz [37]). Liquid deuterium and water have a similar dielectric constant and surface tension. By contrast, the viscosity of deuterium at $25^{\circ} \mathrm{C}$ is $25 \%$ greater than that of water. The temperature of maximum density $(\mathrm{g} / \mathrm{cc})$ also differs: $3.98^{\circ} \mathrm{C}$ for water and $11.2^{\circ} \mathrm{C}$ for deuterium. This difference can make the liquid deuterium effects similar to cooling or apparent temperature effects in experiments. However, the greater difference (except mass) between the isotopes is in self-ionization: liquid deuterium's self-dissociation is five times smaller than that of water, or its $\mathrm{pH}$ at $25^{\circ} \mathrm{C}$ is 7.41 instead of 7.0. We expected to find only slight apparent temperature and viscosity effects on our experiments. Instead, we observed accelerated excitability collapse in both systems (Klink et al. [38]; Fernandes de Lima and Hanke [12]). In contrast to the lower excitability and the fast pathway toward collapse in bulk reactors, the optical profiles of individual B-Z oscillations were not much affected, suggesting that the kinetics of the reaction system was much less affected than the global coupling within the system (Klink et al. [38]). Among the unexpected results was a potential drop at the reference electrode with amplitude of the same order of magnitude as that for the retinal waves (see Fig. 4 of Fernandes de Lima and Hanke [12]).

We used silver wire coils covered with silver chloride, these electrodes are nicknamed "nonpolarizable" because they tend to have a stable interfacial potential with the surrounding solution. The experimenter sets this potential as the relative "zero potential" in the recording system. We could see that as soon as the deuterium solution reached the bottom of the Petri dish that contained the eyecup, there was a linear potential drop at the reference electrode with no measurable latency. When the deuterium solution was again replaced by water, there was complete recovery of the interfacial potential. This result and the parallel roads to collapse in B-Z and retinas were baffling, but might be explained if water can store charge. It turned out that it can. We refer to the experiments of Gerald Pollack and Fernando Galembeck (Ovchinnikova and Pollack [50]; Santos et al. [56]).

We also became aware of the exclusion zone (EZ) found in interfacial water close to polyelectrolytes and its implication for the physiology of biological membranes and of blood flow in venules and capillaries (Zheng and Pollack [78]; Zheng et al. [77]; Gouveia and Galembeck [26]; Chai and Pollack [6]; Bunkin et al. [3]; Rohani and Pollack [53]). And finally, in 2014 in a Gerald Pollack seminar at Galembeck's institute, we became aware of the polar liquid bridges studied by Elmar C. Fuchs' group (see Fuchs et al. [19] and Wexler et al. [74] or better watch their videos ${ }^{2}$ ). The electrical energy stored in

\footnotetext{
${ }^{2}$ In the videos shown in the paper in the Journal of Visual Experiments (JoVE), all the features of the transition from quiescence to steady-state far from equilibrium are shown in the water bridges. These features are common to all dissipative structures; not only water but also other polar liquids form bridges. We quote... "A number of common solvents can form such bridges as well as low conductivity solutions and colloidal suspensions.”... More recent results are presented and discussed in the last Water Conference (2016) in this free-access video: https://www.youtube.com/watch?v=-mB9pLSWHFI. It is the last
} 
dissociated interfacial water can be expended in the forms of heat, non-Planck infrared radiation and coherent slow flow of charged dissociated water. With this energy in mind, we could now reinterpret our own previous experimental results as well as those of others (see also Fernandes de Lima and Hanke [14]). For example, in 1993 unequivocal coherent flow was demonstrated in a B-Z system (Rovinsky and Menziger [54]). The authors filled to the brim a glass cylinder of $3.2 \mathrm{~mm}$ diameter and $25 \mathrm{~cm}$ length with cation exchanger beads of $40 \mu \mathrm{m}$ diameter that were loaded with the catalyst ferroin and filled the remaining space (30\% of the total volume) with the reaction solution. In order to simplify the mathematics, they treated their system as one-dimensional, but in reality it provided a good analogy for interacting membranes within the neuropil where cation-exchanging external leaflets of membranes interact. Rovinsky and Menziger interpreted their data in terms of an activator/inhibitor model with differential flow of chemical species. We propose that, within the dissociated interfacial water, two types of flow coexist: a fast free-proton flow and a slow flow of hydronium ions analogous to the flows demonstrated in water bridges (Fuchs et al. [19]) and nafion interfaces (a polyelectrolyte, sulfate anions polyacid created by DUPONT) (Rohani and Pollack [53]). Therefore, their system is not a chemical system but an electrochemical one. The same is true of the B-Z systems in general; otherwise the liquid deuterium effects would not make sense. In summary: what brought about the puzzling parallel behavior that we observed for 15 years in B-Z and in vitro retinas (Hanke et al. [29]; Wiedemann et al. [75]; Klink et al. [38]; Fernandes de Lima and Hanke [12]) is the presence of the energy of dissociated water, which provides the crucial clue for understanding self-organization in these systems.

\section{The basement membrane and extracellular matrix as charged gels or smart gels}

To the best of our knowledge, the first paper to address the role of polyelectrolytes in nerve excitation was authored by Tasaki et al. [71]. They found that, if most of the cytoplasm is extruded or sucked out and the axon tube filled with inorganic solutions, excitability could be maintained for several hours, showing that excitability is a property of the membrane, not the cytoplasm. Furthermore, they found that the ions could be ordered in relation to favorability of excitation and that this sequence just followed the Hofmeister series or lyotropic series of colloidal physical chemistry (see Appendix). In 1967 Victor E. Shashoua, under the explicit (mentioned in the paper) influence of Aharon Katzir Katchalsky, made the first attempt to construct an artificial axon using polyelectrolytes (Shashoua [57]). A direct descendant of this line of research is Gary Wnek's quest for the artificial axon (Wnek [76]).

Here a digression is necessary to introduce the asymmetry of biological membranes. These are composed of two lipid leaflets, each incorporating two different polyelectrolyte gels (for more details see Fernandes de Lima and Hanke [14]). The external leaflet incorporates glycolipids (gangliosides) that have sialic acid "trees" protruding at 90 degrees to the plane of the membrane. These are exclusive components of the external leaflet. All receptors, channels and transporters are glycoproteins and also have the same "trees" of acidic anions protruding from the lipid bilayer toward the extracellular matrix. Along the membrane plane we have the glycosaminoglycans (GAGs) heparan sulfate, hyaluronan and associate proteins. From the physico-chemical point of view, we have two polyanionic gels able to dissociate water. This structure generates the strong trans-membrane electrical field that astonished physicists

presentation of the day, and begins 4 hours and 10 minutes after the start of the video. The two kinds of protons found in the bridge and the state of water between ice and liquid are discussed. At the questions time, Elmar Fuchs admits on the importance of the geometry of the dishes for the stability of the bridges. Geometry is an important factor for the propagation of waves in excitable media. 
(estimated at $100 \mathrm{kV} / \mathrm{cm}$ see, for example, Frölich [18]). This field created by the structure, stabilizes the structure, a feature common in excitable media that Hermann Haken called "circular causality". The internal gel is of course the cytoskeleton.

Although the cytoskeleton and the external leaflet of the biological membrane (this gel being continuous with the extracellular matrix) differ in composition, they have in common the property of phase transitions or "volume phase transitions" (see Appendix) in which the heteropolymer contracts and the water and ionic atmosphere around the polymer change. For example cytoplasm extracted from Xenopus oocytes can undergo several cycles of contraction/expansion with temperature (Ezzel et al. [10]; Field et al. [17]). The actomyosin heteropolymer responds to $\mathrm{pH}$ and calcium but can have these cycles even in conditions of very low calcium concentration (Ezzel et al. [10]). Katchalsky called this bulk (macroscopic) behavior mechano-electrical coupling and discussed its implication for cell physiology (Katchalsky [35,36]).

Again, the pioneer in exploring volume phase transitions of polyelectrolytes in the context of nerve excitation was Ichigi Tasaki (Tasaki and Byrne [67,68]; Tasaki [64,65]). He also measured the liberation of heat at wave onset both in action potentials and retinal spreading depressions (Tasaki and Iwasa [70]; Tasaki and Byrne [66]) phenomena that we now associate with relaxation of structured interfacial water involved in the polyelectrolyte response to changes induced by electrical and/or mechanical stimulation. In axons and the heart these waves are physiological whereas in retina and cortex they are not, and are therefore associated with pathophysiological events. The same dynamics that produces waves produces other types of macroscopic electrochemical patterns: standing patterns and self-sustained sequences of spirals (Dahlem and Müller [8]; Fernandes de Lima and Hanke [11,14,15]). These patterns can be associated with "petit mal" seizures, complex seizures perceptual distortions and transient global amnesia that either impair consciousness or distort perception.

\section{A single physico-chemical mechanism explains apparent different effects of salts}

In their 1965 paper, Tasaki et al. state clearly that the "pores" concept and the equivalent circuit membrane model of Hodgkin and Huxley could not predict some of his results. Hence, the physical chemical interpretation or macromolecular approach is an alternative explanation for excitability in membranes and tissue. If Tasaki's membrane is assumed, then we can have an alternative interpretation for excitability modulation effects of lithium salts for example. Lithium was the least favorable cation for axon excitability. The sequence found was $\mathrm{Rb}>\mathrm{K}>\mathrm{NH}_{4}>\mathrm{Na}>\mathrm{Li}$. The affinity of alkali metal ions for the carboxyl groups in gels is $\mathrm{Li}<\mathrm{Na}<\mathrm{K}$ (Tasaki et al. [71]). If lithium depressed excitability in the one-dimensional action potential due to lyotropic series effects, then the same effect is expected in the two-dimensional retinal waves and the three-dimensional heartbeat. We have a report that dietary lithium salts slow the propagation of cortical spreading depression waves (Guedes et al. [27]). There is a positive association between the amplitudes of $V$ and $d V / d t$ and the spread velocity of retinal spreading depression waves $\left(r^{2}=0.88\right)$ (Peixoto [51]). Therefore, spread velocity and excitability are associated in spreading depression waves. In other words, lithium depressed the excitability of the cortex in rats. Finally, lithium salts alter the electrocardiogram and in rare cases life-threatening side effects have been reported with ventricular arrhythmias. The depression of $T$ wave amplitude and prolongation of $Q T$ interval are the more common ECG repercussions in patients using lithium (Mateer and Clark [42]; Simard et al. [61]; Hsu et al. [31]; Mohandas and Rajmohan [44]). The increase of $Q T$ interval is compatible with a prolongation of the absolute refractory period of heart fibers. In each heartbeat every fiber goes 
through a sequence of states: quiescent, excited and refractory. The temporal sequence within the population of fibers is coupled, such that every fiber state sequence is locked to the overall tissue pattern. When a patch of tissue changes its absolute refractory period, it falls out of phase with the tissue and it can be the origin of extra systoles and at the limit, ventricular chaotic scrolls will interact in deadly ventricular arrhythmias.

Lithium lyotropic series effects can explain reported consequences of the use of lithium on one and three dimensions of excitation waves. These effects are due to the ionic atmosphere around the negative charges of the polyacids. When this atmosphere changes, the structure changes as well as the electric field associated with the structure (remember the circular causality). In other words the energy of the gel changes. We did not find reports of lithium effects on retinal spreading depression waves; however, besides the rodent cortical wave effects referred to above, the "favorability" position of $\mathrm{Na}$ in the sequence found in axons, is the next after $\mathrm{Li}$. It happens that $\mathrm{Na}$ has a strong effect on retinal waves. If the concentration of $\mathrm{NaCl}$ in the perfusion solution of the retina increases from $100 \mathrm{mM}$ to $140 \mathrm{mM}$, the amplitude of the potential drop associated with retinal waves $(V)$ is depressed to $40 \%$ of the controls, $d V / d t$ peak to $23 \%$ of controls and spread velocity to $66 \%$. Besides, $\mathrm{NaCl}$ drastically changes the retinal transparency and inverts the intrinsic optical signal of spreading depression waves (Peixoto [51]) suggesting marked structural changes within the tissue. With Tasaki's physico-chemical approach we can explain lithium salt effects in excitable tissue with a single explanation.

That the most common therapeutic use of lithium is in psychiatry, we do not think is just a coincidence, but a clue to where mental functions dwell. If one takes into account that when the external gel of membranes changes, all membrane intrinsic proteins will have to change, all active and passive transporters will change. These changes will be reflected in their kinetics. All biochemical effects of lithium salts can be explained by a physico-chemical structural change at membranes.

\section{The energy state of smart gels and a non-linear polyelectrolyte synapse}

The tight coupling between energy state and shape found in charged gels could be explored by nature as a cognitive mechanism. The same Victor Shashoua referred to above, who demonstrated the excitability of a polyelectrolyte synthetic membrane, later discovered an extracellular glycoprotein, ependymin, associated with learning in fish, mice and rats (Shashoua [58]; Shashoua and Schmidt [60]; Shashoua et al. [59]). He proposed that the ability of this protein to facilitate heteropolymer combination in the matrix, and its modulation by calcium, contributed to neural plasticity. However, the first researchers to attribute memory mechanisms to metastable states of polyelectrolytes were Eberhard Neumann and Aharon Katchalsky in the early seventies of last century. We quote: “... the potential change (of action potentials) corresponds to a variation of the field intensity from $-70 \mathrm{kV} / \mathrm{cm}$ to $+50 \mathrm{kV} / \mathrm{cm}$. We may assume that these electric fields act not only across the nerve membrane, but also in its close proximity and probably across the synaptic junction... We may therefore consider (metastable) conformational changes of biopolymers induced by electric impulses as a model reaction for the process of imprinting nerve impulses in the structures involved in the physical memory..." (Neumann and Katchaslky [49]). Another quote: "Controlled changes in the environment of metastable macromolecular structures or subcellular macromolecules, organizations such as membranes by high electric fields or by ion gradients can induce conformational changes which could serve as reproducible imprints of a memory nature" (Neumann and Katchalsky [48]). 
In 1977, experiments with the basement membrane of striated muscle showed a kind of long-term memory (two weeks): If both the muscle fiber and nerve terminal were allowed to degenerate, the pattern of the neuromuscular junction was still imprinted on the polyelectrolyte gel as demonstrated by showing that any newly formed nerve terminal or fiber membrane expressed junctional proteins at the exact place occupied by the analogous earlier structures (Marshall et al. [41]; Sanes et al. [55]; Ide et al. [32]; Jo and Burden [34]). The basement membrane is both a supramolecular arrangement and an energy pattern associated with the supramolecular architecture. Imaging this gel with infrared cameras it can be expected to show several resonances interlacing at different, superposed spatial and temporal scales. These resonances will show the effects of mechanical (sound and ultrasound) and electromagnetic energy. In order to see them one should use infrared cameras to see the non-Planck infrared radiation from the dissociated water associated with the gel. Disturbing the energy pattern can alter this memory and either the neuromuscular junction proteins will be expressed at random sites or not at all. A clear prediction.

In the field of experimental cortical spreading depression, the term "functional ablation" was used in order to show the reversible loss of the integrative cortical function when awake animals had cortical waves elicited. Impaired learning and memory and blockade of inter-hemispheric transfer of learning were demonstrated mainly by Olga and Jan Bures in Czechoslovakia (see Bures and Buresova [5]) and later by other groups. For reviews see Bures et al. [4] and Gallo [23]. The abrupt and reversible structural change at wave onset releases energy, part of which is expressed in the form of heat (Tasaki and Byrne [66]). We think that most of this heat is produced by the loss of structured interfacial water referred to in previous sections.

The hallmarks of histological findings in Alzheimer's disease are the heteropolymers made between a part of an intrinsic membrane glycoprotein, $\beta$ amyloid protein, and glycosaminoglycans (GAGs) among other extracellular matrix components. These heteropolymers are called senile plaques and can be large $(50 \mu \mathrm{m})$. The neuronal death marked by the fibrillary tangles inside neurons could be a consequence of the loss of glial/neural interaction. We could see the power of protamine heteropolymers in the basement membrane and the excitability control of axons, retinas and heart as discussed in previous sections (Fernandes de Lima et al. [16]). In our experiments, protamine brought excitability collapse without apparent interference in the endfeet membrane mechanisms. A mechanical clamping of the gel appears to be the cause of the hampering of wave spread, and excitability collapse.

Basement membranes, glycocalix and extracellular matrix are all names of charged gels or polyelectrolytes. They make up the interfaces of excitable tissue and mediate glial/neural dynamics. This supramolecular arrangement can express macroscopic electrochemical patterns at supracellular dimensional scales. These patterns can be physiological, as with heartbeats and action potentials, or pathophysiological as shown by some consequences of spreading depression waves which may be either solitary or arranged in self-sustaining sequences. Standing patterns may also be dissipative electrochemical structures. The symptoms associated with these emergent patterns have perceptual and cognitive manifestations and may have repercussions for a range of other mental functions. Spreading depression waves capture essential features of glial/neural non-linear dynamics, which are themselves important to many aspects of neural, and thus 'mental', function.

\section{Concluding remarks}

This short essay offers an outline of the history of concepts and empirical research on glial-neuronal non-linear dynamics. Irreversible thermodynamics provides a useful theoretical framework for under- 
standing many aspects of brain function. Eberhard Neumann and Aharon Katchalsky's polyelectrolyte (and hence non-linear) synapses and Ichigi Tasaki's excitable membrane model are essential to this framework. However, we are barely beginning to understand the energy and structure of dissociated interfacial water. How it relates to ions is maybe the key to finally understanding the Hofmeister series and why we have the precise distribution of cation and anion chaotropes and kosmotropes in excitable tissue (see Appendix). The usefulness of excitable media and physical-chemical concepts derived from irreversible thermodynamics is shown clearly in their applicability to systems as diverse as both heart and brain.

\section{Acknowledgements}

The authors wish to thank Dr. Chris Nunn for his help with the manuscript and useful discussions. We are also thankful for the kindness and generosity of this special issue editor, Reinoud Maex.

\section{Appendix}

Figure 1 shows Aharon Katchalsky's concept of how the ionic atmosphere around polyelectrolytes could influence their overall shape. The transition from the extended to the collapsed state is often

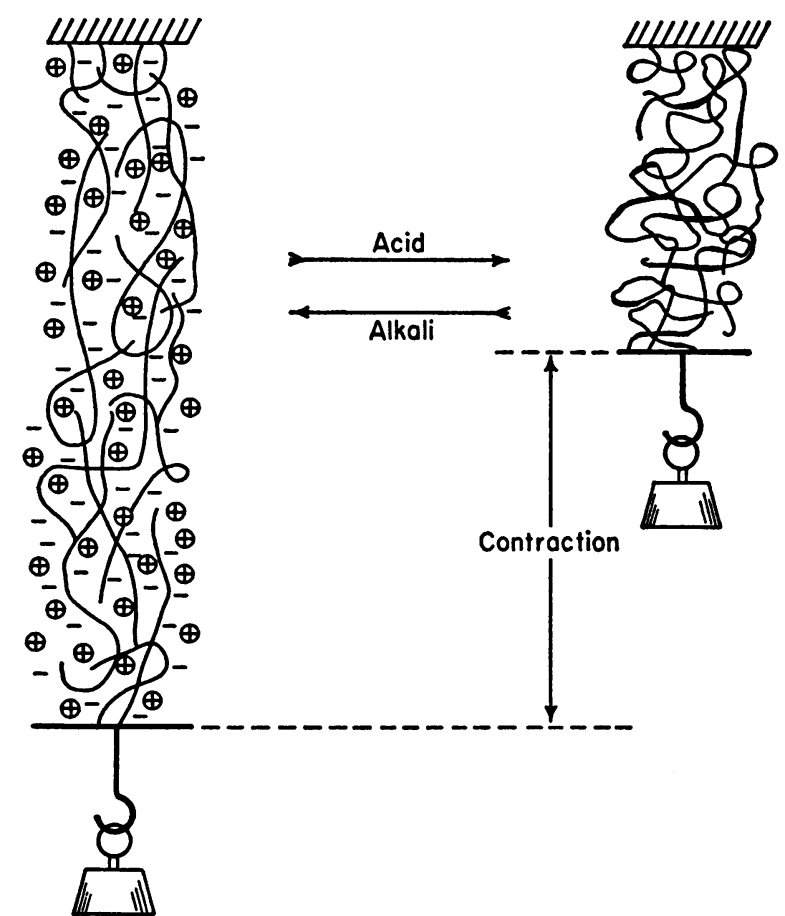

Polyocid gel in sodium

Polyacid gel in acid solution: hydroxide solution: expanded controcted; weight is lifted

Fig. 1. The figure shows the influence of the ionic atmosphere around polyelectrolytes in changing their shape. Any change in the local degree of ionization causes a corresponding change in the overall electrical field. This change in turn is expressed in the change in dimensions or in contractile force. Aharon Katchalsky called this behavior "mechano-electrical coupling". He proposed that nature could use this property of polyelectrolytes in many cell functions. Modified from Katchalsky [35]. 
abrupt in charged gels, the physical chemists call these transitions "volume phase transitions" observed in artificial (Tanaka [63]) as well as biological polyelectrolytes. One example, upon exocytosis from goblet cells, granules of mucin undergo a 600-fold growth in volume in seconds (Verdugo [73]).

Volume phase transitions of the external gel associated with the lipid bilayer are the key to Ichigi Tasaki's excitable membrane model. In his own words: "The cortical gel layer of nerve fibers has the properties of a cation exchanger. Hence, this layer can, and actually does, undergo a reversible abrupt structural change when monovalent cations (e.g. $\mathrm{Na}^{+}$) are substituted for the divalent counter-ions (e.g. $\mathrm{Ca}^{2+}$ )"... "the reversible abrupt structural changes occurring in the cortical gel layer of nerve fibers are at the base of the process of excitation and conduction. There is abundant evidence to show that these structural changes are associated with divalent-monovalent cation exchanges taking place in the negatively charged sites in the cortical gel layer" (Tasaki [65]). The "two states" swollen and collapsed gel model for the action potential can best be modelled by the FitzHugh-Nagumo two-variable mathematical description of the action potential.

The first publication about the possible involvement of macromolecular arrangements and their behavior in the action potential is the 1965 Tasaki et al. publication. They found that the favorability for the maintenance of excitability of several cations and anions followed the Hofmeister series. Originally this series shows the qualitative ranking of ions in the precipitation of solutions of hen egg white. Franz Hofmeister published his series in 1888 (Hofmeister [30]), the same series is also named lyotropic series. According to their ability to stabilize and make soluble proteins, ions are said to be Kosmotropes $\left(\mathrm{Na}^{+}\right.$, salting out) or Chaotropes $\left(\mathrm{K}^{+}\right.$, salting in).

Tasaki and collaborators found that depending on the salt used in perfused axons, excitability could be a question of minutes or several hours and that the ranking order of the cations and anions followed the Hofmeister series, suggesting the involvement of polyelectrolytes in nerve excitation.

\section{References}

[1] A. Araque, V. Parpura, R.P. Sanzguiri and P.G. Haydon, Tripartite synapses: Glia, the unacknowledged partner, Trends in Neurosciences 22 (1999), 208-215. doi:10.1016/S0166-2236(98)01349-6.

[2] R. Birks, B. Katz and R. Miledi, Physiological and structural changes at the amphibian myoneural junction, in the course of nerve degeneration, J. Physiol. 150 (1960), 145-168. doi:10.1113/jphysiol.1960.sp006379.

[3] N.F. Bunkin, V.S. Gorelik, V.A. Kozlov, A.V. Shkirin and N.V. Suyazov, Colloidal crystal formation at the "Nafion-water" interface, J. Phys. Chem. B 118 (2014), 3372-3377. doi:10.1021/jp4100729.

[4] J. Bures, E. Buresova and J. Krivanek, The Mechanism and Applications of Leão's Spreading Depression of Electroencephalographic Activity, Academic Press, New York, 1974.

[5] J. Bures and O. Buresova, Cortical spreading depression as a memory disturbing factor, J. Comp. Physiol. Psychol. 56 (1963), 268-272. doi:10.1037/h0045749.

[6] B. Chai and G.H. Pollack, Solute-free interfacial zones in polar liquids, J. Phys. Chem. B 114 (2010), 5371-5375. doi:10. 1021/jp100200y.

[7] J.A.A. Da Silva, P. Spencer, M.A. Camillo and V.M. Fernandes de Lima, Gyroxin and its biological activity: Effects on CNS basement membranes and endothelium and protease-activated receptors, Curr. Med. Chem. 19 (2012), 281-291. doi:10.2174/092986712803414123.

[8] M. Dahlem and S.C. Müller, Self-induced splitting of spiral shaped spreading depression waves in chicken retina, Exp. Brain Res. 115 (1997), 319-324. doi:10.1007/PL00005700.

[9] S. Dierig, Extending the neuron doctrine: Carl Ludwig Schleich (1859-1922) and his reflections on neuroglia at the inception of the neural-network concept in 1894, Trends in Neurosciences 17 (1994), 449-452.

[10] R. Ezzel, A.J. Brothers and W.Z. Cande, Phosphorylation-dependent contraction of actomyosin gels from amphibian eggs, Nature 306 (1983), 620-622. doi:10.1038/306620a0.

[11] V.M. Fernandes de Lima and W. Hanke, Excitation waves in central grey matter: The retinal spreading depression, Progress in Eye and Retinal Research 16(4) (1997), 657-690. doi:10.1016/S1350-9462(96)00038-9. 
[12] V.M. Fernandes de Lima and W. Hanke, Modulation of CNS excitability by water movement. The $\mathrm{D}_{2} \mathrm{O}$ effects on the non-linear neuron-glial dynamics, J. Biophys. Chem. 2 (2011), 253-260.

[13] V.M. Fernandes de Lima and W. Hanke, The kinetics of non-synaptically triggered acute excitotoxic responses in the central nervous system observed using intrinsic optical signals, CNS Neurol. Disorders Drug Target 11 (2012), 132-141. doi: $10.2174 / 187152712800269704$.

[14] V.M. Fernandes de Lima and W. Hanke, Macroscopic self-organized electrochemical patterns in excitable tissue and irreversible thermodynamics, Open Journal of Biophysics 6 (2016), 98-124. doi:10.4236/ojbiphy.2016.64011.

[15] V.M. Fernandes de Lima, J.R.C. Piqueira and W. Hanke, The tight coupling and non-linear relationship between the macroscopic electrical and optical concomitants of electrochemical CNS waves reflect the non-linear dynamics of neural glial propagation, Open Journal of Biophysics 5 (2015), 1-20. doi:10.4236/ojbiphy.2015.51001.

[16] V.M. Fernandes de Lima, P. Spencer and W. Hanke, Interaction of small cationic peptides with intact basement membranes. A study using intrinsic optical signals of chick retinas, Curr. Med. Chem. 21(12) (2014), 1458-1466. doi:10.2174/ 092986732112140319102212.

[17] C.M. Field, M. Wühr, G.A. Anderson, H.Y. Kueh, D. Strickland and T.J. Mitchison, Actin behavior in bulk cytoplasm is cell cycle regulated in early vertebrate embryos, J. Cell Sci. 124 (2011), 2086-2095. doi:10.1242/jcs.082263.

[18] H. Frölich, The extraordinary dieletrics properties of biological materials and the action of enzymes, Proceedings of the National Academy of Sciences of the USA 72 (1975), 4211-4215. doi:10.1073/pnas.72.11.4211.

[19] E.C. Fuchs, B. Bitschnau, A.D. Wexler, J. Woisetschläger and F.T. Freund, A quasi-elastic neutron scattering study of the dynamics of electrically constrained water, J. Phys. Chem. B 119 (2015), 15892-15900. doi:10.1021/acs.jpcb.5b10751.

[20] R. Galambos, A glia-neural theory of brain function, Proceedings of the National Academy of Sciences of the USA 47(1) (1961), 129-136. doi:10.1073/pnas.47.1.129.

[21] R. Galambos, Robert Galambos in: The History of Neuroscience in Autobiography, Vol. 1, L.R. Squire, ed., Society for Neurosciences, Washington, DC, 1996, pp. 178-220.

[22] R. Galambos, Reflections on the conceptual origins of neural-glial interactions, Neuron Glia Biology 3 (2007), 89-91.

[23] M. Gallo, Reversible inactivation of brain circuits in learning and memory research, in: Neural Plasticity and Memory: From Genes to Brain Imaging, F. Bermúdez-Rattoni, ed., CRC Press/Taylor \& Francis, Boca Raton, FL, 2007, Chapter 8. Available from: http://www.ncbi.nlm.nih.gov/books/NBK3922.

[24] P. Glansdorff and I. Prigogine, Thermodynamic Theory of Structure, Stability and Fluctuations, Wiley-Interscience, London, 1971. ISBN 0-471-30280-5.

[25] N.A. Gorelova and J. Bureš, Spiral waves of spreading depression in the isolated chicken retina, J. Neurobiol. 14 (1983), 353-363. doi:10.1002/neu.480140503.

[26] R.F. Gouveia and F. Galembeck, Electrostatic charging of hydrophilic particles due to water adsorption, J. Am. Chem. Soc. 131 (2009), 11381-11386. doi:10.1021/ja900704f.

[27] R. Guedes, L.F. Amorin, M.C. Medeiros, A.T. Silva and M.R. Teodósio, Effect of dietary lithium on cortical spreading depression, Braz. J. Med. Biol. Res. 22 (1989), 923-925.

[28] R.W. Guillery, Relating the neuron doctrine to the cell theory. Should contemporary knowledge change our view of the neuron doctrine?, Brain Res. Rev. 55 (2007), 411-421. doi:10.1016/j.brainresrev.2007.01.005.

[29] W. Hanke, M. Wiedemann and V.M. Fernandes de Lima, Control of the excitability of neuronal tissue by weak external forces, Faraday Discuss. 120 (2002), 237-248. doi:10.1039/b102706a.

[30] F. Hofmeister, Zur Lehre von der Wirkung der Salze, Arch. Exp. Pathol. Pharmakol. (Leipzig) 24 (1888), 247-260. doi:10. 1007/BF01918191.

[31] C.-H. Hsu, P.-Y. Liu, J.-H. Chen, T.-L. Yeh, H.-Y. Tsai and L.-J. Lin, Electrocardiographic abnormalities as predictors for over-range lithium levels, Cardiology 103 (2005), 101-106. doi:10.1159/000082471.

[32] C. Ide, K. Thyama, R. Yokota, T. Nitatory and S. Onoder, Schwan cell, basal lamina and muscle regeneration, Brain Res. 288 (1983), 61-75. doi:10.1016/0006-8993(83)90081-1.

[33] K. Iguchi, Motoyosi Sugita - A "widely unknown" Japanese thermodynamicist who explored the 4th law of thermodynamics for creation of the theory of life, Open Journal of Biophysics 6 (2016), 125-232. doi:10.4236/ojbiphy.2016. 64012.

[34] S.A. Jo and S. Burden, Synaptic basal lamina contains a signal for synaptic specific transcription, Development 115 (1992), 673-680.

[35] A.K. Katchalsky, Polyelectrolytes and their biological interactions, Biophys. J. 4 (1964), 9-41. doi:10.1016/S00063495(64)86924-1.

[36] A.K. Katchalsky, Biological flow structures and their relation to chemo-diffusional coupling, Neuroscience Res. Prog. Bull. 9 (1972), 397-413.

[37] J. Katz, Chemical and biological studies with deuterium, American Scientist 48(4) (1960), 544-580.

[38] O. Klink, W. Hanke, E. Gerbershagen and V.M. Fernandes de Lima, Influence of heavy water in the Belouzov-Zabotinsky reaction, in: Wave Propagation in Materials for Modern Applications, A. Petrin, InTech, Vukovar, Croatia, 2010. ISBN 978-953-7619-65-7. 
[39] K.S. Lashley, Patterns of cerebral integration indicated by the scotomas of migraine, Arch. Neurol. Psych. 46 (1941), 331-339. doi:10.1001/archneurpsyc.1941.02280200137007.

[40] A.A.P. Leão, Spreading depression of activity in the cerebral cortex, J. Neurophysiol. 7 (1944), 359-390.

[41] L.M. Marshall, J.R. Sanes and U.J. MacMahan, Reinnervation of original synaptic sites on muscle fiber basement membrane after disruption of the muscle cells, Proceedings of the National Academy of Sciences of the USA 74 (1977), 3073-3077. doi:10.1073/pnas.74.7.3073.

[42] J.R. Mateer and M.R. Clark, Lithium toxicity with rarely reported ECG manifestations, Annals of Emergency Medicine 11 (1982), 208-211. doi:10.1016/S0196-0644(82)80500-3.

[43] P.M. Milner, Note on a possible correspondence between the scotomas of migraine and spreading depression of Leão, EEG and Clin. Neurophysiol. 10 (1958), 705. doi:10.1016/0013-4694(58)90073-7.

[44] E. Mohandas and V. Rajmohan, Lithium use in special populations, Indian J. Psychiatry 49 (2007), 211-218. doi:10.4103/ 0019-5545.37325.

[45] S. Mori, W.H. Miller and T. Tomita, Microelectrodes study of spreading depression (SD) in the frog retina. General observation of field potentials associated with SDs, Jpn. J. Physiol. 26 (1976), 203-217. doi:10.2170/jjphysiol.26.203.

[46] S. Mori, W.H. Miller and T. Tomita, Microelectrode study of spreading depression (SD) in frog retina - Müller cell activity and $\left[\mathrm{K}^{+}\right]$during SD, Jpn. J. Physiol. 26 (1976), 219-233. doi:10.2170/jjphysiol.26.219.

[47] S. Mori, W.H. Miller and T. Tomita, Müller cell function during spreading depression in the frog retina, Proceedings of the National Academy of Sciences of the USA 73 (1976), 1351-1354. doi:10.1073/pnas.73.4.1351.

[48] E. Neumann and A.K. Katchalsky, Hysteretic conformational changes in biopolymers induced by high electrical fields. Model for a physical record of biological memory, in: Proc. Eur. Biophys. Congr. (Baden, Austria), Vol. 6, E. Broda, ed., Verlag Wiener Med. Akad., Vienna, Austria, 1971, pp. 91-96.

[49] E. Neumann and K. Katchalsky, Long-lived conformation changes induced by electric impulses in biopolymers, Proceedings of the National Academy of Sciences of the USA 69 (1972), 993-997. doi:10.1073/pnas.69.4.993.

[50] K. Ovchinnikova and G.H. Pollack, Can water store charge?, Langmuir 25 (2009), 542-547. doi:10.1021/la802430k.

[51] N.L.V. Peixoto, A depressão alastrante na retina, Master's thesis, Universitade Estadual de Campinas, 1997 (in Portuguese).

[52] I. Prigogine and R. Lefevre, Symmetry breaking instabilities in dissipative systems, J. Chem. Phys. 48 (1968), 1695-1700. doi:10.1063/1.1668896.

[53] M. Rohani and G.H. Pollack, Flow through horizontal tubes submerged in water in the absence of a pressure gradient: Mechanistic considerations, Langmuir 29 (2013), 6556-6561. doi:10.1021/la4001945.

[54] A.B. Rovinsky and M. Menzinger, Self-organization induced by the differential flow of activator and inhibitor, Phys. Rev. Lett. 70 (1993), 778-781. doi:10.1103/PhysRevLett.70.778.

[55] J.R. Sanes, L.M. Marshall and U.J. MacMahan, Reinnervation of muscle fiber basal lamina after removal of muscle fibers, J. Cell Biol. 78 (1978), 176-198. doi:10.1083/jcb.78.1.176.

[56] L.S. Santos, T.R.D. Ducati, L.B.S. Balestrin and F. Galembeck, Water with excess electric charge, J. Phys. Chem. C 115 (2011), 11226-11232. doi:10.1021/jp202652q.

[57] V. Shashoua, Electrically active polyelectrolyte membrane, Nature 215 (1967), 846-847. doi:10.1038/215846a0.

[58] V.E. Shashoua, Ependymin, a brain extracellular glycoprotein, and CNS plasticity, Ann. NY Acad. Sci. 627 (1991), 94114. doi:10.1111/j.1749-6632.1991.tb25916.x.

[59] V.E. Shashoua, G.W. Hesse and B. Milinazzo, Evidence for the in vivo polymerization of ependymin: A brain extracellular glycoprotein, Brain Res. 522 (1990), 181-190. doi:10.1016/0006-8993(90)91460-X.

[60] V.E. Shashoua and R. Schmidt, Learning and memory: Neurochemical aspects, in: Encyclopedia of Neurosciences, G. Adelman, ed., Birkhauser Boston, 1987, pp. 577-579.

[61] M. Simard, B. Gumbiner, A. Lee, H. Lewis and D. Norman, Lithium carbonate intoxication. A case report and review of the literature, Arch. Intern. Med. 149 (1989), 36-46. doi:10.1001/archinte.1989.00390010054004.

[62] E. Sugaya, M. Takato and Y. Noda, Neuronal and glial activity during spreading depression in cerebral cortex of cat, J. Neurophysiol. 38 (1975), 822-841.

[63] T. Tanaka, Phase transitions of gels, in: Polyelectrolyte Gels, R. Harlan, ed., American Chemical Society, Washington, DC, 1992, pp. 1-21. doi:10.1021/bk-1992-0480.ch001.

[64] I. Tasaki, Evidence for phase transition in nerve fibers, cells and synapses, Ferroelectrics 220 (1999), 305-316. doi:10. 1080/00150199908216221.

[65] I. Tasaki, On the reversible abrupt structural changes in nerve fibers underlying their excitation and conduction processes, in: Phase Transitions in Cell Biology, G.H. Pollack and W.C. Chin, eds, Springer, Science-Business, 2008, pp. 1-21. doi:10.1007/978-1-4020-8651-9_1.

[66] I. Tasaki and P.M. Byrne, Demonstration of heat production associated with spreading depression in the amphibian retina, Biochemical and Biophysical Research Communications 102 (1991), 293-297. doi:10.1016/0006-291X(91)90519-D.

[67] I. Tasaki and P.M. Byrne, Discontinuous volume transitions in ionic gels and their possible involvement in the nerve excitation process, Biopolymers 32 (1992), 1019-1023. doi:10.1002/bip.360320812. 
[68] I. Tasaki and P.M. Byrne, Discontinuous volume transitions induced by calcium-sodium ion exchange in anionic gels and their neurobiological implications, Biopolymers 34 (1994), 209-215. doi:10.1002/bip.360340207.

[69] I. Tasaki and J.J. Chang, Electric response of glia cells in cat brain, Science 128 (1958), 1209-1210. doi:10.1126/science. 128.3333.1209.

[70] I. Tasaki and Iwasa, Temperature changes associated with nerve excitation: Detection by using polyvinylidene fluoride film, Biochemical and Biophysical Research Communications 101 (1981), 172-176. doi:10.1016/S0006291X(81)80026-5.

[71] I. Tasaki, I. Singer and T. Takenaka, Effects of internal and external ionic environment on squid giant axon. A macromolecular approach, J. Gen. Physiol. 48 (1965), 1095-1123. doi:10.1085/jgp.48.6.1095.

[72] T. Tomita, Spreading depression potential (SDP) in frog retina, Ann. Acad. Bras. Cienc. 56 (1984), 505-517.

[73] P. Verdugo, Goblet cell hydration kinetics of exocytosed mucins in cultured secretory cells of the rabbit trachea: A new model, CIBA Found. Symp. 109 (1984), 212-225.

[74] A.D. Wexler, M. López Sáenz, O. Schreer, J. Woisetschläger and E.C. Fuchs, The preparation of electrohydrodynamic bridges from polar dielectric liquids, J. Vis. Exp. 91 (2014), e51819.

[75] M. Wiedemann, V.M. Fernandes de Lima and W. Hanke, Gravity dependence of waves in the retinal spreading depression and in gel type Belousov-Zhabotinsky systems, Phys. Chem. Chem. Phys. 4 (2002), 1370-1373. doi:10.1039/b109166m.

[76] G.E. Wnek, Perspective: Do macromolecules play a role in nerve stimulation and nervous transmission?, J. Polym. Sci. Part B: Polym. Phys. 54 (2016), 7-14. doi:10.1002/polb.23898.

[77] J.M. Zheng, W.C. Chin, E. Khijniak, E. Khijniak Jr. and G.H. Pollack, Surfaces and interfacial water: Evidence that hydrophilic surfaces have long-range impact, Adv. Colloid Interface Sci. 127 (2006), 19-27. doi:10.1016/j.cis.2006.07. 002.

[78] J.M. Zheng and G.H. Pollack, Long-range forces extending from polymer-gel surfaces, Phys. Rev. E Stat. Nonlin. Soft Matter Phys. 68(3) (2003), 031408. doi:10.1103/PhysRevE.68.031408.

[79] V.S. Zykov, Excitable media, Scholarpedia 3 (2008), 1834. doi:10.4249/scholarpedia.1834. 